\title{
Fusion of Gene Regulatory and Protein Interaction Networks Using Skip-Chain Models
}

\author{
Iti Chaturvedi ${ }^{1}$ and Jagath C. Rajapakse ${ }^{1,2,3}$ \\ ${ }^{1}$ Bioinformatics Research Center, School of Computer Engineering, \\ Nanyang Technological University, Singapore \\ 2 Singapore-MIT Alliance, Singapore \\ ${ }^{3}$ Department of Biological Engineering, Massachusetts Institute of Technology, USA
}

\begin{abstract}
Inference of Gene Regulatory Networks (GRN) is important in understanding signal transduction pathways. This involves predicting the correct sequence of interactions and identifying all interacting genes. Using only gene expression data is insufficient, so additional sources of data like protein-protein interaction network (PPIN) are required. In this paper, we model time delayed interactions using a skip-chain model which finds missing edges between non-consecutive time points based on PPIN. Highest Viterbi approximation is used to select skip-edges. The $k$-skip validation model checks for $k$ missing genes between a predicted interaction, giving us advantages of validation as well as expansion of GRN. The method is demonstrated on a cell-division cycle data of S.cerevisiae (yeast). Comparison of the present method, with a previous approach of modeling PPIN by using a Gibbs prior are given.
\end{abstract}

Keywords: Dynamic Bayesian networks, Gene Regulatory networks, Higher-order Markov chains, Protein-Protein interactions, Viterbi algorithm.

\section{Introduction}

Most processes of signal transduction involve ordered sequences of biochemical reactions inside the cell, which are carried out by an ensemble of enzymes activated by secondary messengers, resulting in signal transduction pathways. The DNA in a cell contains genes which are converted to mRNA(expressed genes) through transcription and then translated into proteins. Consequently, signal transduction pathways are often interpreted in terms of gene regulatory networks (GRN) and protein-protein interaction networks (PPIN). High throughput techniques allow generation of both gene and protein interaction data simultaneously. Studies that use both gene and protein expressions have been mostly devoted to a single type of data while the other type of data is restricted to validation [1, 2, 3]. Using a single data source of interactions has its own limitations and could create errors in the analysis of the interactome. This is due to two main reasons: firstly, both microarray and PPI data have a lot of noise due to measurement errors, varied transcriptional response in the cell and inter-functional phenomena. Secondly, complex formation and other critical interactions that regulate biological processes take place

M. Chetty, A. Ngom, and S. Ahmad (Eds.): PRIB 2008, LNBI 5265, pp. 214 224, 2008.

(C) Springer-Verlag Berlin Heidelberg 2008 
at the protein level. A protein being a product of a gene, a joint mining of gene regulatory networks and protein interaction networks could reveal genes that are co-expressed and their proteins also interact. Clusters and interactions found by joint mining will be more reliable than those found by using only one type of data. We may thereby have high confidence of finding gene clusters that are regulated by the same mechanism and belong to the same biological process.

Fusion of GRN and PPIN has been attempted by using Naive Bayes where genes are partitioned into different pathways [1]. Likelihood of the data becomes higher when genes in the same pathway have similar expression profiles and are interacting. The unified model is learned using Expectation Maximization algorithm. This method however requires the user to specify the number of pathways which is often unknown. Cross-graph mining [2] can achieve integration by looking for partial cliques in both GRN and PPIN simultaneously. A weighted score of SVM classification of gene expression and functional classification of PPI was suggested in [3. The weights are determined by simulated annealing. Prior modeling of PPIN into Bayesian learning has been done using Gibbs distribution 4. A Gibbs random field equivalent to a first-order Markov random field is used to represent the prior graph. This approach is insufficient because many timedelayed interactions are known to exist.

In this paper, we extend skip-chain sequence models [5] and use Viterbi approximation of dynamic Bayesian networks (DBN) to include time delayed interaction edges based on PPIN data. The method is demonstrated by using yeast cell cycle data, where genes are differentially expressed in each phase. Genes in one phase regulate by activation/inhibition genes in the next phase resulting in a cycle. A comparison is done with prior modeling of PPIN using Gibbs distribution. The method almost doubles the sensitivity and is robust to the increase in number of genes. The paper is organized as follows. Section 2 discusses Bayesian networks (BN), dynamic Bayesian networks(DBN) and their extension to higherorder. Section 3 explains the different models: we first discuss prediction of GRN using skip-chain models and our extension to fuse prior knowledge of PPIN. Next we describe the $k$-skip validation model of GRN based on PPIN. In section 4 , we demonstrate our approach on 5 different datasets from yeast cell cycle. A comparison is done with prior modeling of PPIN using Gibbs distribution. Lastly, we make our conclusions in section 5 .

\section{Modeling GRN with Higher-Order Bayesian Networks}

Microarray experiments simultaneously measure expression patterns of thousands of genes over different experimental conditions or over time. Let us consider a set of $n$ such genes $G=\left\{g_{1}, g_{2}, \ldots, g_{n}\right\}$, and time-series gene expression data of length $m$ for all the genes. Let the microarray data matrix $X=\left[x_{1}, x_{2} \ldots x_{n}\right]^{T}$ in which row vector $x_{i}=\left(x_{i, 1}, x_{i, 2}, \ldots, x_{i, m}\right)$ corresponds to gene expression time series of gene $g_{i}$ where $x_{i, t}$ denotes the expression level at time $t$. Let the set of parents (or genes regulating) of gene $i$ be denoted as $a_{i}$ and the number of states the nodes in $a_{i}$ take be $q_{i}$. 


\subsection{Bayesian Networks (BN)}

A BN is a graphical model that can represent a joint multivariate probability distribution by capturing the properties of conditional independencies among the variables. It is a directed acyclic graph (DAG) having a structure $S$ and a set of conditional distributions. BN can appropriately model the genes as nodes in the network and edge as causal interaction's between them.

The Bayesian network decomposes the joint probability of genes into the product of conditional probabilities by using the chain rule and independence of non-descendant genes, given their parents

$$
p\left(x_{1}, x_{2}, \ldots, x_{n}\right)=\prod_{i=1}^{n} p\left(x_{i} \mid a_{i}, \theta_{i}\right)
$$

where $p\left(x_{i} \mid a_{i}, \theta_{i}\right)$ is the conditional probability of gene expression $x_{i}$ given its parents, and $\theta_{i}$ denotes the parameters of the conditional probabilities.

The optimal structure is obtained by maximizing the posterior probability for $S$. From Bayes theorem,

$$
\max _{S} p(S \mid X)=\max _{S} p(S) p(X \mid S)
$$

where $p(S)$ is the prior probability of the network structure. Given the set of conditional distributions with parameters $\theta=\left\{\theta_{i} \mid i=1,2, \ldots n\right\}$, the likelihood can be written as

$$
p(X \mid S, \theta)=\int p(X \mid S, \theta) p(\theta \mid S) d \theta
$$

Let us assume that gene expressions carry discrete levels of gene expression $: x_{i, t}=k$ where $k \in\{1,2, \ldots, d\}$ and $d$ denotes the maximum level of expression of any gene. Let $\theta_{i j k}=p\left(x_{i, t}=k \mid a_{i}=j\right)$ and $N_{i j k}$ be the number of instances of $\theta_{i j k}$ that occur in the training data. Using the property of decomposability,

$$
p(X \mid S, \theta)=\prod_{i=1}^{n} \prod_{j=1}^{q_{i}} \prod_{k=1}^{d} \theta_{i j k}^{N_{i j k}}
$$

Assuming global and local parameter independence,

$$
p(\theta \mid S)=\prod_{i=1}^{n} p\left(\theta_{i} \mid S\right)=\prod_{i=1}^{n} \prod_{j=1}^{q_{i}} p\left(\theta_{i j} \mid S\right)=\prod_{i=1}^{n} \prod_{j=1}^{q_{i}} \prod_{k=1}^{d} p\left(\theta_{i j k}\right)
$$

Substituting Eq. (4) and Eq. (5) into Eq. (3) gives

$$
p(X \mid S)=\prod_{i=1}^{n} \prod_{j=1}^{q_{i}} \int \prod_{k=1}^{d} \theta_{i j k}^{N_{i j k}} p\left(\theta_{i j k}\right) d \theta_{i j k}
$$


We can approximate the integral by using maximum likelihood estimate known as Bayesian Information criterion (BIC) [6]. We can estimate $\theta_{i j k}$ as

$$
\theta_{i j k}=\frac{N_{i j k}}{\sum_{k=1}^{d_{i}} N_{i j k}}
$$

Taking the log-likelihood, gives us the following expression:

$$
B I C=\log p(X \mid S, \theta)=\sum_{i=1}^{n} \sum_{j=1}^{q_{i}} \sum_{k=1}^{d} N_{i j k} \log \frac{N_{i j k}}{\sum_{k=1}^{d_{i}} N_{i j k}}
$$

Hence the likelihood approximation of the score needs no prior over parameters as the posterior probability captures information of the prior. The likelihood approximation is known to be good when using large amounts of data. However, if the dataset is small it will over-penalize.

The acyclic condition in BN does not allow self regulation and feedback, which are characteristic of GRN. To overcome this, dynamic Bayesian networks (DBN) are used in which a transition network from one time point to the next characterizes the GRN.

\subsection{Dynamic Bayesian Networks (DBN)}

A first-order dynamic Bayesian network (DBN) is defined by a pair of structures $\left(S_{t}, S_{t+1}\right)$ corresponding to time instances $t$ and $t+1$ and a transition network of interactions between the two networks. The DBN structure is obtained by unrolling the transition network over time (Figure 10. In slice $t$, the parents of $x_{i, t}$ are those specified in the initial network $S_{t}$, and in slice $t+1$, the parents of $x_{i, t}$ are those genes in slice $t$ corresponding to parents of $x_{i, t}$ in $S_{t}$. The transition network of interactions between time instances $t$ and $t+1$ is given by

$$
p\left(x_{i, 1}, \ldots, x_{i, m}\right)=\prod_{t=1}^{m} p\left(x_{i, t} \mid x_{i, t-1}, \theta_{i}\right)
$$

where $t=0$ corresponds to the dummy initial state.

The metric for a DBN can hence be defined as

$$
p(X \mid S, \theta)=\prod_{t=1}^{m} \prod_{i=1}^{n} \prod_{j=1}^{q_{i}} \prod_{k=1}^{d} \theta_{i j k}^{N_{i j k}^{(t, t+1)}} \forall t
$$

where $N_{i j k}^{(t, t+1)}$ correspond to the transition network $\left(S_{t}, S_{t+1}\right)$. The first-order DBN has $2 n$ nodes.

The first-order Markov DBN can be extended to a higher-order to allow higher-order interactions among variables. For an $r$-order Markov DBN, given a node $x_{i}$, its parents are chosen from the set of variables $X[t-r] \cup X[t-r-$ $1] \ldots X[t-1]$, where $X[t]$ is a column of gene expression matrix at time $t$. We assume $r$-order stationary Markov chain. With this assumption an $r$-order transition network has $(r+1) \times n$ nodes (Figure 1), where $n$ is number of genes. 


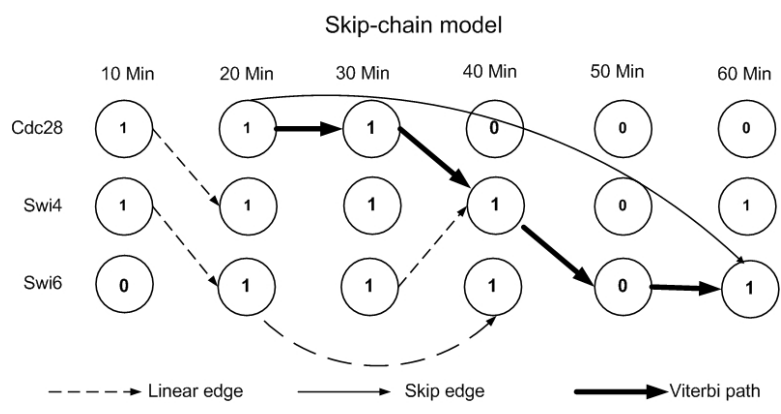

Fig. 1. Illustration of six time points and three genes of G1-S phase of cell-cycle in a dynamic Bayesian network. The dashed edges are linear $r=1,2$ order edges found by linear features. The solid directed edge is an example of skip-edge over four time points which models a long-distant dependency. The bold directed line shows a skip path computed by Viterbi algorithm.

Now $N_{i j k}$ can include cases from any of the previous $r$ time slices. As $r$ increases the search space becomes extremely large. In order to address this problem we propose skip-chain sequence models.

\section{Fusion of GRN and PPIN}

\subsection{Skip-Chain Sequence Model}

A higher-order DBN is unable to accomodate long-distance dependencies because the number of parameters increases with the order. For example, if order is $r$, for binary gene expressions with a maximum $q$ parents, there will be $r^{q}$ parameters for each gene. To overcome the explosion of parameters, a skip-chain sequence model 7] augments a linear chain with skip-features that represent long range dependencies. It then simply factorizes the prediction probability into linear and skip features. The number of skip-features can be implemented based on prior knowledge as given in PPIN.

Linear-chain feature functions $f_{u}\left(x_{i}, a_{i(t-r: t)}, t\right)$ represent local dependencies that are consistent with an $r$-order Markov assumption of gene expressions. These represent dependencies between nearby time points and cannot represent higher-order dependencies like activation, inhibition or feedback, which occur throughout the time-series of the pathway. We relax the above assumption by using skip-chain feature functions $g_{u^{\prime}}\left(x_{i}, a_{i}, s_{t}, t\right)$ which exploit dependencies between genes that are arbitrarily distant at time instances $s_{t}$ and $t$ respectively. Such a skip-feature models variable length Markov chain upto $m-1$ order where $m$ is number of time points. The score of the structure is a weighted sum of linear and skip-edge scores:

$$
\log p\left(x_{i} \mid a_{i}\right) \propto \sum_{u=1}^{U} \lambda_{u} f_{u}\left(x_{i}, a_{i(t-r: t)}, t\right)+\sum_{u^{\prime}=1}^{U^{\prime}} \mu_{u^{\prime}} g_{u^{\prime}}\left(x_{i}, a_{i}, s_{t}, t\right)
$$

where $\lambda_{u}$ and $\mu_{u^{\prime}}$ are weights for corresponding features. 
Inspired by a previous application of skip-chain models to summarise group meetings [5], we achieve fusion by designing an interaction skip feature $\left(g_{i}, g_{j}\right.$, $\left.s_{t}, t\right)$ between genes $g_{i}$ and $g_{j}$, having similar expressions at two different time points $s_{t}$ and $t$ where $s_{t} \in\{1,2,3, . ., t-2\}$. We further constrain that these genes must be interacting as indicated by the PPIN. In the next section, we discuss how a Viterbi approximation is used to determine the optimal skip features.

\subsection{Skip-Edge Determination}

In the skip-chain models, higher-order interactions are represented by skip-edges. We used Viterbi approximation of a DBN [8] to determine the optimal skipfeatures, as the maximum a posterior forward path through the trellis. This lets us compare different time-delayed skip-features between two genes. An example of such a path to model a fourth order interaction is shown in Figure 1. The Viterbi algorithm first calculates log transition probabilities from the data. The transition probability from gene $g_{j}, t-1$ to $g_{i}, t$ at is defined as

$$
p\left(x_{i, t} \mid x_{j, t-1}\right)=\frac{n_{i, j, t}}{\sum_{j^{\prime}=1}^{n} n_{i, j^{\prime}, t}}
$$

where $n_{i, j, t}$ denotes number of occurrences where $x_{i, t}=x_{j, t-1}=1$ in the discretized gene expression data.

Then, forward state transitions $l^{t}$ that give minimum transition probability at each time point is chosen:

$$
l^{t}=\arg \min _{j} p\left(x_{i, t} \mid x_{j, t-1}\right)
$$

And the skip-edge score is the negative of the total transition probabilities at the last time point:

$$
g\left(x_{i}, a_{i}, s_{t}, t\right)=-\sum_{t^{\prime}=s_{t}}^{t} \log l^{t}
$$

where the parent set $a_{i}$ has only one gene at time point $s_{t}$. As there can be many time-delayed skip features between two genes, we choose the time delay which has the highest normalized a posterior probability. Normalization is done by dividing the total probability by length of skip-edge.

The linear feature model is obtained from the Bayesian network:

$$
f\left(x_{i}, a_{i(t-r: t)}, t\right)=\log p\left(x_{i} \mid a_{i}, \theta_{i}\right)
$$

where $a_{i}$ is parent set from any of previous $r$ time points. The score of the structure now becomes

$$
\log p\left(x_{i} \mid a_{i}\right) \propto f\left(x_{i}, a_{i(t-r: t)}, t\right)+\mu g\left(x_{i}, a_{i}, s_{t}, t\right)
$$


Since we have a single parameter $\mu$, it can be simply determined by repeated trial.

In Figure 1 the edges of three genes in the first six time points can be viewed. The dashed edges are linear $r=1,2$ order edges. The solid directed edge is an example of skip-edge over three time points which models a long-distant dependency.

\section{3 $k$-Skip Validation}

Validation of GRN is often done with the use of experimentally verified interactions listed in corresponding PPIN. It is presumed that probabilistic influence flows in the graph and genes $g_{i}$ and $g_{j}$ maybe connected if there is an unblocked path $g_{i} \rightarrow g_{1} \rightarrow g_{2} \rightarrow \ldots \rightarrow g_{k} \rightarrow g_{j}$ in the PPIN. Since PPI data available is undirectional, the true structure of $S$ is predicted by using a $k$-skip validation model [9]. The $k$-skip validation model looks for one or more genes that are skipped when predicting an interaction.

Consider two genes, $g_{i}$ and $g_{j}$, interacting in the GRN, The $k$-skip validation model checks for a cascade of $k$ genes $\left(g_{1}, g_{2}, \ldots, g_{k}\right)$ such that any gene $g_{c}$ in the cascade interacts with the next gene $g_{c+1}$ where $c \in\{1,2, . ., k-1\}$. Lastly $g_{i}$ must interact with $g_{1}$ and $g_{j}$ to interact with $g_{k}$. Some examples are shown in Figure 2. Interaction Cln3-Cdc28 is predicted using microarray data and is also found in PPIN; Mbp1-Cln3 is not found in PPIN, however a 1-skip form Mbp1-Swi4-Cln3 exists in PPIN; Similarly a 2-skip form of Exg2-Htb2 will be Exg2-Hsp82-Spt15-Htb2.

A DBN chooses parent-child relations with the highest likelihood based on the time-series microarray data and assumes a first-order Markov chain. However, many time-delayed interactions are known to exist and this causes skipping of genes. The $k$-skip validation could expand a predicted GRN as well as correct using information from PPIN. It has been previously reported that 1-skip and 2-skip forms are common in predicted GRN.

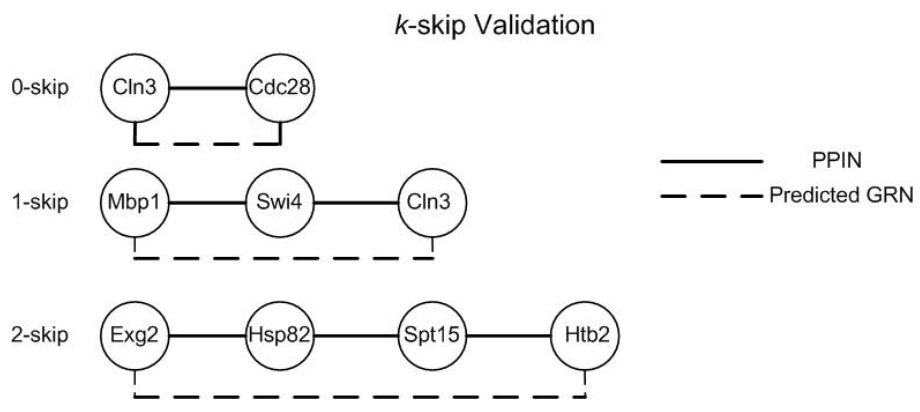

Fig. 2. $k$-skip validation model looks for one or more genes in the PPIN that were skipped while predicting gene interaction. Dashed lines are predicted false positives of GRN and solid lines are PPIN. 


\section{Results and Discussion}

We evaluated our method on a dataset acquired in cell-cycle regulation of yeast [10. The microarray dataset was taken from Spellman et al. 10] which has mRNA measurements of 6,178 genes under different experimental settings. The expression values range from -2 to +2 , which were normalized and discretized into levels 0 (under-expression) and 1 (over-expression), respectively. We chose 24 time points of cdc-15 cell cycle arrest ranging from 10 to $290 \mathrm{~min}$. Yeast cell-division cycle consists of four main phases: genome duplication (S phase), and nuclear division (M phase), separated by two gap phases (G1 and G2). The S-G1-M-G2-S form a cycle for cell duplication. Phase specific gene expression profiles were extracted based on the list given in 10. The PPIN dataset for validation was downloaded from BioGRID (Biological General Repository for Interaction Datasets) [1]. It has over 50,000 experimental as well as literaturederived interactions for the genome. Phase specific prior PPIN for the pathway was derived from the validation set. Using phase specific PPIN network and gene expression profiles, we looked for skip-edges.

For expression data discretized into two levels 0 (under-expressed) and 1(overexpressed), we consider 7 time points (9 to 16) of peak activity for 118 genes in G1 phase of cell-cycle. Table 1 shows that a skip feature captures the correlation among non-consecutive time points. A chi-square test shows that consecutive time points $t$ and $t-1$ were not significantly correlated with a p-value 0.77 , while noncosecutive time points $s_{t}$ and $t$ where $s_{t} \in\{1,2,3, \ldots, t-2\}$ are correlated with a p-value less than 0.001 . It can be seen that there are 9146 skip-edges.

A genetic algorithm (GA) was implemented to find the optimal structure of the instantaneous network of GRN [12]. A solution individual $C=\left\{c_{i j}\right\}_{n \times n}$ where $c_{i j} \in\{0,1, \ldots, r\}$ denotes the strength of the interaction between genes $i$ and $j$, and 0 means no interaction. We used highest time-delay as the order of an interaction in PPIN and a random order for unknown interactions. Each individual in the GA allowed upto three parents for a gene. The GA chooses the network with best combination of skip and linear edges (Eq. 16). Simulation was done at different numbers of individuals $(\mathrm{N})$ and generations $(\mathrm{G})(\mathrm{N}=200 / 300 / 400$ and

Table 1. Contingency tables for 118 genes in G1 phase, at peak activity (time points 9 to 16) for yeast cell cycle data. Chi-square test shows that the adjacent expressions $x_{i, t}$ and $x_{j, t-1}$ are not correlated with p-value $=0.77$, however expressions at far away time points $x_{i, t}$ and $x_{j, s t}$ where $s_{t} \in\{1,2,3, \ldots, t-2\}$ clearly influence each other with a p-value $\leq 0.001$.

\begin{tabular}{|c|c|c|c|c|}
\hline & $x_{i, t}=0$ & $x_{i, t}=1$ & Chi-square & P-value \\
\hline Linear edge & & & & \\
$x_{j, t-1}=1$ & 518 & 1954 & 0.08 & $p=0.77$ \\
$x_{j, t-1}=0$ & 152 & 554 & & \\
\hline Skip edge & & & 96.46 & $p \leq 0.001$ \\
$x_{j, s_{t}}=1$ & 2848 & 9146 & & \\
$x_{j, s_{t}}=0$ & 2512 & 10918 & & \\
\hline
\end{tabular}



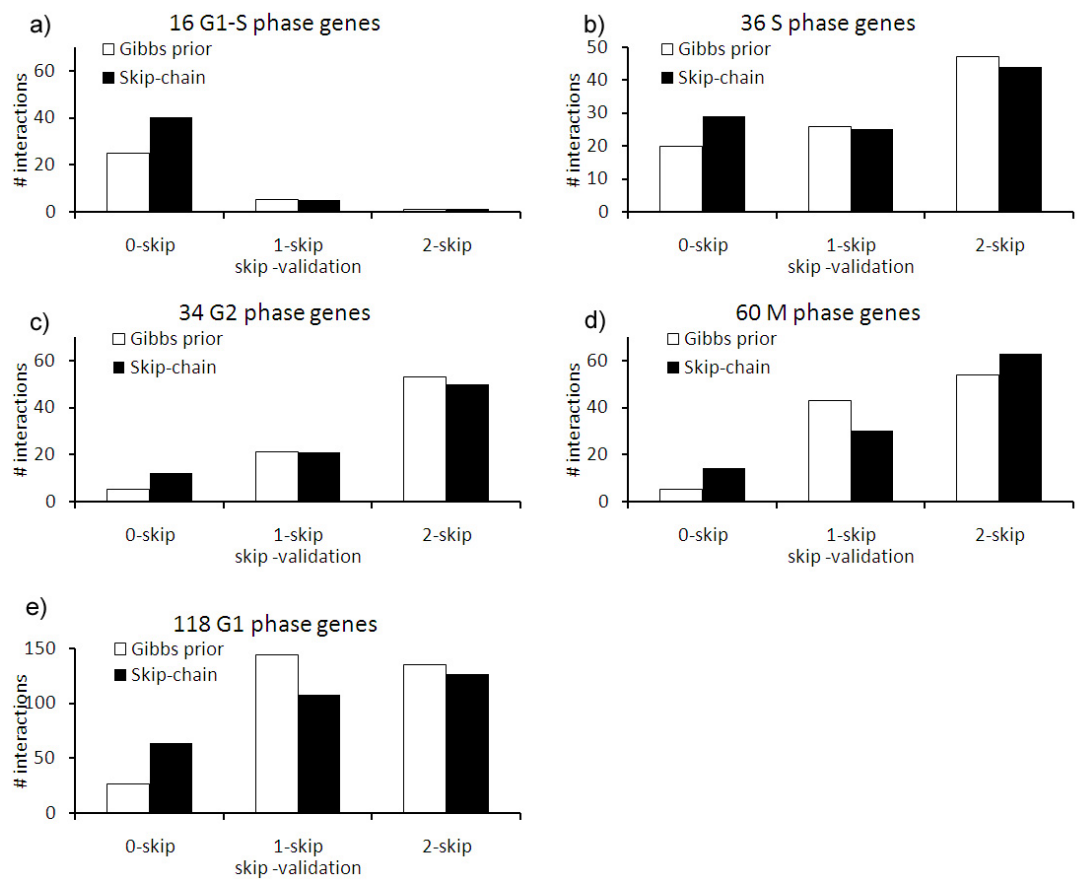

Fig. 3. $k$-skip validation to compare Gibbs prior and skip-chain models on five datasets in yeast cell-cycle: (a) 16 genes from G1-S phase (b) 36 genes from S phase (c) 34 genes from G2 phase (d) 60 genes from M phase and (e) 118 genes from G1 phase. Number of correct predictions by skip-chain is almost twice those predicted by Gibbs prior.

$\mathrm{G}=300 / 400 / 500$ ). The GA stops when the maximum number of generations is reached or if the score does not change for 20 consecutive generations. The best prediction among all five runs was considered.

Previously, PPIN have been fused with GRN using Markov random field as the prior network [4. The interaction potential for a PPIN validated interaction is defined by the user. The entropy $P(S)$ then becomes a sum of interaction potentials for a predicted GRN. Appropriate weights of the PPI edge in Gibbs random field and skip-chain DBN which gave best prediction were got by repeated trial. The appropriate weight for 16 genes with 4678 skip-edges was found to be 40 , as further increase in weight did not improve prediction. We approximately used 80 for 36 genes, 160 for 60 genes and so on.

The above procedure was carried out for five sets of genes (i) 16 genes in G1-S phase (ii) 36 genes in S phase (iii) 34 genes in G2 phase (iv) 60 genes in $\mathrm{M}$ phase and (v) 118 genes in G1 phase (Figure 3). For all five datasets our method outperformed the previous approach of fusing PPIN as a Gibbs prior. The sensitivity approximately doubles in all cases while the specificity remains high (Table 2). We conclude that the method is robust to the increase in the number of genes. Unlike Gibbs prior, the skip-chain model consider's 
Table 2. Comparison of fusion of GRN and PPIN for Yeast cell cycle data by using a Gibbs prior and skip-chain model

\begin{tabular}{|c|c|c|c|c|}
\hline & \multicolumn{2}{|c|}{ Gibbs prior } & \multicolumn{2}{c|}{ Skip-chain model } \\
\hline Number of Genes & Sensitivity & Specificity & Sensitivity & Specificity \\
\hline 16 G1-S & 0.35 & 0.73 & 0.65 & 0.93 \\
36 S & 0.59 & 0.86 & 0.74 & 0.88 \\
34 G2 & 0.34 & 0.88 & 0.99 & 0.99 \\
60 M & 0.1 & 0.9 & 0.3 & 0.9 \\
118 G1 & 0.1 & 0.95 & 0.21 & 0.96 \\
\hline
\end{tabular}
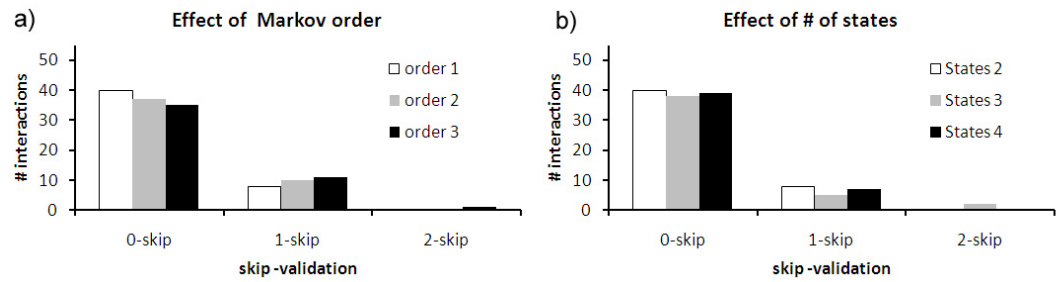

Fig. 4. $k$-skip validation of a predicted GRN for 16 G1-S phase genes of Yeast cell cycle (a) Increasing the order of a linear edge decreases the number of correct predictions.

(b) Increasing the number of discrete states does not show any significant change in prediction performance.

the time delayed interaction between two genes. It is also able to distinguish between different interactions while Gibbs prior assigns equal weights to all PPIN interactions. We only found 1-skip or 2-skip cascades and no 3-skip cascades were found in all the datasets. The number of 2-skip was also higher in the larger networks (see Figure 3 ).

A few interactions were seen outside the prior network, suggesting missing members of a pathway. Increasing the Markov order of the DBN causes overfitting, this could be because of redundant effects of skip-chain and the higher-order DBN(Figure 4a). Here we have considered binary states $\{0,1\}$ to represent under expression and over expression of a gene. Increasing the number of states did not give a significant increase in performance (Figure $4 \mathrm{~b}$ ). It might however be useful when applied to other datasets with higher noise.

\section{Conclusion}

Higher-order dependencies are significant for time-series gene expression data analysis when deriving gene regulatory networks. We propose a method for effective fusion of GRN and PPIN by introducing skip-edges found on PPIN into GRN predicted by first-order DBN modeling. This almost doubles the sensitivity of GRN, compared to the earlier modeling using Gibbs distribution while the number of false positives remains same. 
Feature functions are a good way to include prior knowledge into a generegulatory network. The method is found to be robust when applied to larger networks. The approach is computationally efficient. Here, we have only considered interactions where both genes have similar expression at different time points. Similar modeling can be done for activation, inhibition and feedback events of causal networks of genes.

\section{References}

1. Segal, E., Wang, H., Koller, D.: Discovering molecular pathways from protein interaction and gene expression data. Bioinformatics 19(suppl. 1), i264-i272 (2003)

2. Pei, J., Jiang, D., Zhang, A.: Mining Cross-graph Quasi-cliques in Gene Expression and Protein Interaction Data. In: Proceedings of the 21st International Conference on Data Engineering (ICDE 2005), vol. 5(8), pp. 353-356 (2005)

3. Tu, K., Yu, H., Li, Y.-X.: Combining gene expression profiles and protein-protein interaction data to infer gene functions. Journal of Biotechnology 124, 475-485 (2006)

4. Nariai, N., Kim, S., Imoto, S., Miyano, S.: Using protein-protein interactions for refining gene networks estimated from microarray data by Bayesian networks. PSB. 9, 336-347 (2004)

5. Galley., M.: A Skip-Chain Conditional Random Field for Ranking Meeting Utterances by Importance. In: Proceedings of the 2006 Conference on Empirical Methods in Natural Language Processing (EMNLP 2006), pp. 364-372 (2006)

6. Friedman, N., Murphy, K., Russell, S.: Learning the structure of dynamic probabilistic networks. In: Fourteenth Conf. on Uncertainty in Artificial Intelligence (UAI), pp. 139-147 (1998)

7. Sutton, C., McCallum, A.: Collective segmentation and labeling of distant entities in information extraction. In: Presented at ICML Workshop on Statistical Relational Learning and Its Connections to Other Fields, University of Massachusetts (2004)

8. Tang, H., Huang, T.S.: Improved graphical model for audiovisual object tracking. In: IEEE International conference on Multimedia and Expo. pp. 997-1000 (2006)

9. Chaturvedi, I., Sakharkar, M.K., Rajapakse, J.C.: Validation of Gene Regulatory Networks from Protein-Protein Interaction Data: Application to Cell-cycle Regulation. In: Rajapakse, J.C., Schmidt, B., Volkert, L.G. (eds.) PRIB 2007. LNCS (LNBI), vol. 4774, pp. 300-310. Springer, Heidelberg (2007)

10. Spellman, P.T., Sherlock, G., Zhang, M.Q., et al.: Comprehensive Identification of Cell Cycle-regulated Genes of the Yeast Saccharomyces cerevisiae by Microarray Hybridization. Molecular Biology of the Cell 9, 3273-3297 (1998)

11. Stark, C., Breitkreutz, B.J., Reguly, T., et al.: BioGRID: a general repository for interaction datasets. Nucleic Acids Res. 34(Database issue), D535-539 (2006)

12. Xing, Z., Wu, D.: Modeling Multiple Time Units Delayed Gene Regulatory Network Using Dynamic Bayesian Network. In: Sixth IEEE International Conf. on Data Mining (ICDMW 2006), pp. 190-195 (2006) 